\title{
De-Bruijn-Folgen und Zauberei
}

\author{
Martina Rahija $\cdot$ Annegret Weng
}

Eingegangen: 12. Oktober 2020 / Angenommen: 23. November 2020 / Online publiziert: 21. Dezember 2020

(C) Der/die Autor(en) 2020

Zusammenfassung De-Bruijn-Folgen in der Zauberkunst wurden bereits von mehreren Autoren vorgeschlagen und auch mit Erfolg auf der Bühne eingesetzt (siehe z. B. [2, 4, 11]). Insbesondere gibt es dazu einen bekannten effektvollen Trick mit einem Skatspiel bestehend aus 32 Karten. Dieser setzt nicht voraus, dass sich der Zauberer die De-Bruijn-Folge und die Kodierung in Kartenwerte merken muss, da er alle notwendigen Informationen berechnen kann.

In diesem Artikel gehen wir auf die mathematischen Grundlagen spezieller, durch lineare Abbildungen generierte De-Bruijn-Folgen ein. Mit der Theorie gelingt es uns, den Trick von 32 Karten auf das Pokerspiel mit 52 Karten bzw. auf das Kartenspiel „Set“ mit 81 Karten zu übertragen. In beiden Fällen kann sich der Zauberer mit etwas Übung die nötigen Informationen errechnen und muss dadurch wenig memorieren.

\section{Einleitung}

Ein Zauberer hält die folgenden 8 Karten in der Hand:
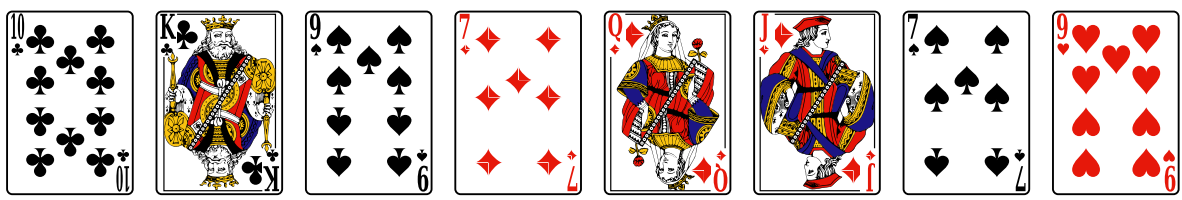

Unter Beibehaltung der Reihenfolge bildet er ein bildunten gehaltenes Päckchen mit der Kreuz 10 als oberste Karte, gefolgt vom Kreuz König, Pik 9 usw. Er gibt das Kartendeck an einen Zuschauer weiter und bittet ihn, ein paar Mal abzuheben und

M. Rahija $\cdot$ A. Weng $(\triangle)$

Hochschule für Technik, Schellingstr. 24, 70174 Stuttgart, Deutschland

E-Mail: annegret.weng@hft-stuttgart.de 
sich anschließend die oberste Karte zu nehmen. Anschließend soll der Zuschauer den Kartenstapel an seinen Nachbarn weitergeben, der sich ebenso die oberste Karte nimmt und den Stapel weiterreicht. Auch dessen Nachbar nimmt sich die nun oberste Karte. Der Zauberer lässt sich von den Zuschauern lediglich die Farben (,rot“ oder „schwarz") ihrer Karten nennen und kann dann die genaue Ausprägung der drei Karten (z. B. „Karo Bube, Pik 7, Herz 9“) benennen (vergl. auch [2, 4]).

Dieser Zaubertrick hat eine mathematische Grundlage. Wir betrachten dazu die folgende endliche Folge bestehend aus $8=2^{3}$ Zeichen aus dem Alphabet $\{0,1\}$ :

00010111.

Wenn wir uns diese Folge als geschlossenen Zyklus vorstellen, treten hier alle möglichen Wörter der Länge $n=3$ genau einmal auf. Nach und nach erhalten wir

000, 001, 010, 101, 011, 111, 110, 100 .

Nun steht eine schwarze Karte für eine 0 und eine rote für eine 1. Dann sind die drei Karten durch ihre Farben eindeutig bestimmt.

Folgen mit dieser Eigenschaft werden nach dem Mathematiker Nicolaas Govert de Bruijn benannt.

Definition 1.1 Sei $\Omega$ ein Alphabet mit $k$ verschiedenen Buchstaben. Dann ist eine De-Bruijn-Folge der Ordnung $n$ über $\Omega$ ein Wort $w$ in $\Omega^{k^{n}}$ mit der Eigenschaft, dass jedes mögliche Wort in $\Omega^{n}$ als Teilwort von $w$ auftritt.

Es gibt zahlreiche Arbeiten über die Konstruktion von De-Bruijn-Folgen (für einen Übersichtsartikel siehe z.B. [5]). Bereits 1934 beschrieb Martin [9] einen einfachen, gierigen Algorithmus zur Konstruktion einer De-Bruijn-Folge und zeigte damit insbesondere die Existenz für beliebiges $k \in \mathbb{N}$ und $n \in \mathbb{N}$. Genauer ist bekannt, dass es für jedes $\Omega$ mit $|\Omega|=k$ und jedes $n \in \mathbb{N}$

$$
\frac{k !^{k^{n-1}}}{k^{n}}
$$

verschiedene De-Bruijn-Folgen gibt [1]. Dabei werden zwei De-Bruijn-Folgen genau dann als gleich angesehen und deshalb nur einfach gezählt, wenn sie durch eine zyklische Transformation ineinander überführt werden können.

De-Bruijn-Folgen haben auch Anwendungen in der Industrie, z. B. bei GPS-Systemen und in der Verschlüsselung [6, 7].

Um einen bühnenwirksamen Zaubertrick zu erhalten, sollte man mehr als 8 Karten verwenden. Praktisch ergeben sich jedoch einige Probleme:

1. Der Zauberer muss in der Lage sein, jeder Farbkombination bzw. jedem Wort der Länge $n$ eine Karte zuzuordnen. Er muss sich also zum einen die Zuordnung zwischen den Wörtern der Länge $n$ und den Karten merken. Zum anderen muss er, um auch die restlichen, von den anderen Zuschauern gezogenen Karten zu 
benennen, entweder die zugrundeliegende De-Bruijn-Folge oder die Karten in der richtigen Reihenfolge im Kopf memorieren.

2. Nach Definition haben De-Bruijn-Folgen über einem Alphabet $\Omega$ mit $k$ Buchstaben immer $k^{n}$ Zeichen für ein $n \in \mathbb{N}$. Die Anzahl der Karten muss aber nicht notwendigerweise eine Potenz sein. So hat das beliebte Pokerkartenspiel 52 Karten. Wie können wir De-Bruijn-Folgen geschickt abschneiden, um eine Teilfolge mit $k^{n-1}<m<k^{n}$ Zeichen zu erhalten, die immer noch die Bedingung erfüllt, dass die Teilwörter der Länge $n$ paarweise verschieden sind?

Diese Fragen werden wir im Folgenden diskutieren. Dabei gehen wir auf die erste Frage in Abschn. 2.2 ein und behandeln die zweite Frage dann in Abschn. 2.3. In Abschn. 3 beschreiben wir konkrete Anwendungen in der Zauberei, einmal für das Pokerkartenspiel mit 52 Karten und für das Setkartenspiel mit 81 Karten.

Die zugrundeliegende mathematische Theorie ist die Theorie der linearen Rekursionen modulo einer Primzahl $p$. Die Mathematik ist in der einschlägigen Literatur gut untersucht (siehe z.B. [6]). Insofern enthält der Artikel keine neuen, mathematischen Erkenntnisse. Wir geben aber eine in sich geschlossene Darstellung, die lediglich Vorkenntnisse über Eigenwerte und -vektoren bzw. zur Diagonalisierbarkeit von Matrizen aus der Linearen Algebra und Eigenschaften endlicher Körper, wie sie etwa in [8] dargestellt werden, voraussetzt.

\section{Linear generierte De-Bruijn-Folgen}

\subsection{Spezialfall $k=2$ und $n=5$}

In [4], Kapitel 2, S. 26 ff wird der in Abschn. 1 beschriebene Zaubertrick durch Betrachtung von De-Bruijn-Folgen der Ordnung 5 über dem Alphabet $\{0,1\}$ auf $32=2^{5}$ Karten, also auf ein typisches Skatkartenspiel, erweitert. Dabei wird eine spezielle Folge verwendet, die es relativ einfach macht, für ein bestehendes Teilwort das nächste Zeichen zu errechnen. Der Vollständigkeit halber beschreiben wir das dort beschriebene Verfahren.

Die zugrundeliegende De-Bruijn-Folge ist

$$
00000100101100111110001101110101 .
$$

Für ein beliebiges fünfstelliges Wort $w_{1} w_{2} w_{3} w_{4} w_{5}$ ergibt sich das nachfolgende (also das um eins nach rechts verschobene) Wort als $w_{2} w_{3} w_{4} w_{5} w_{6}$ mit $w_{6} \equiv w_{1}+$ $w_{3} \bmod 2$. Das fünfstellige Wort 00000 passt nicht in das Schema und wird einfach zwischen 10000 und 00001 eingefügt. Dieses Verfahren erlaubt nun eine einfache Durchführung des Tricks, ohne dass der Vorführende die gesamte Folge memorieren muss.

Bei der Kodierung in Spielkarten bestimmt $w_{1} w_{2}$ die Farbe, z.B. $w_{1} w_{2}=00$ für Kreuz, $w_{1} w_{2}=01$ für Pik, $w_{1} w_{2}=10$ für Herz und $w_{1} w_{2}=11$ für Karo. Das Teilwort $w_{3} w_{4} w_{5}$ legt den Wert der Karte fest: 000 für 7, 001 für 8, 010 für 9, 011 für 10, 100 für Bube, 101 für Dame, 110 für König und 111 für Ass. 
Der Vorführende verteilt nun beispielsweise von links nach rechts die obersten fünf Karten und bittet danach alle Zuschauer mit einer roten Karte aufzustehen. Er muss nun nur noch rechnen. Stehen beispielsweise der 1., 2. und 4. Zuschauer auf, entspricht dies der Binärfolge 11010 und somit ist die Karte des ersten

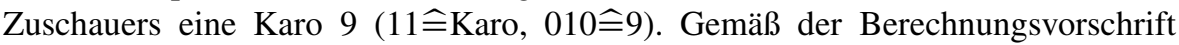
$w_{6}=w_{1}+w_{3} \quad$ mod 2 ergeben sich die nachfolgenden Karten 10101 (Herz Dame), 01010 (Pik 9), 10100 (Herz Bube) und 01000 (Pik 7).

\subsection{Verallgemeinerung}

Diese Vorgehensweise lässt sich verallgemeinern. Dazu stellen wir das Alphabet $\Omega$ durch die Restklassen $\{0,1, \ldots, k-1\}$ modulo $k$ dar. Sei $\mathbb{Z} / k \mathbb{Z}$ der Restklassenring modulo $k$.

Definition 2.1 Eine De-Bruijn-Folge $w=w_{0} \ldots w_{k^{n}-1} \in(\mathbb{Z} / k \mathbb{Z})^{k^{n}}$ heißt linear, wenn es eine Teilfolge $v_{0} \ldots v_{k^{n}-2}:=w_{0} \ldots w_{i-1} w_{i+1} \ldots w_{k^{n-1}}$ und eine $n \times n$-Matrix A über $\mathbb{Z} / k \mathbb{Z}$ der Form

$$
\left(\begin{array}{ccccc}
0 & 1 & 0 & \ldots & 0 \\
0 & 0 & 1 & \ldots & 0 \\
\vdots & \vdots & & \ddots & \vdots \\
0 & 0 & \ldots & & 1 \\
a_{n-1} & a_{n-2} & \ldots & \ldots & a_{0}
\end{array}\right)
$$

gibt, so dass für alle n-stelligen Teilwörter $v_{j} \ldots v_{j+n-1}$

$$
A \cdot\left(v_{j}, \ldots, v_{j+n-1}\right)^{T}=\left(v_{j+1}, \ldots, v_{j+n}\right)^{T}
$$

gilt. Dabei sind die Indizes der $v_{j}$ modulo $k^{n}-1 z u$ verstehen, das heißt, $v_{l}:=$ $v_{l-k^{n}-1}$ für $l \geq k^{n}-1$.

Der Übergang zu der Teilfolge $v_{0} \ldots v_{k^{n}-2}$ in der Definition ist erforderlich, um das $n$-stellige Wort aus lauter Nullen zu eliminieren, das wir durch diese Berechnungsvorschrift nicht generieren können. Es muss wie in Abschn. 2.1 nachträglich an einer passenden Stelle eingefügt werden.

Definition 2.1 können wir auch so formulieren: Eine lineare De-Bruijn-Folge ist eine lineare Rekursion $n$-ter Ordnung modulo $k$.

Im Fall einer linearen De-Bruijn-Folge muss der Zauberer sich nur die Transformation $A$ und die Zuordnung der $k^{n}$ Karten auf $(\mathbb{Z} / k \mathbb{Z})^{n}$ merken. Die in Abschn. 1 und 2.1 beschriebenen Folgen sind linear und lassen sich somit leicht abrufen. Die zugehörigen Matrizen sind hier

$$
\left(\begin{array}{lll}
0 & 1 & 0 \\
0 & 0 & 1 \\
1 & 0 & 1
\end{array}\right) \text { bzw. }\left(\begin{array}{lllll}
0 & 1 & 0 & 0 & 0 \\
0 & 0 & 1 & 0 & 0 \\
0 & 0 & 0 & 1 & 0 \\
0 & 0 & 0 & 0 & 1 \\
1 & 0 & 1 & 0 & 0
\end{array}\right)
$$


Allerdings gibt es auch nicht-lineare De-Bruijn-Folgen. Betrachten wir beispielsweise

$$
0000101100111101 .
$$

Dies ist eine De-Bruijn-Folge der Ordnung 4 über $\Omega=\{0,1\}$. Wir sehen, dass auf die Teilfolge 0110 eine 0 und auf die Teilfolge 1111 eine 0 folgt. Wäre die Folge linear, müsste auf $1001=0110+1111$ ebenfalls eine 0 folgen.

Für eine Matrix $A$ der Form (2), die eine De-Bruijn-Folge erzeugt, muss offensichtlich $a_{n-1} \neq 0$ gelten, da sonst zwei $n$-stelligen Teilwörtern, deren letzte $n-1$ Stellen übereinstimmen, das gleiche $n$-stellige Teilwort nachfolgen würde. Daraus folgt, dass $A$ invertierbar ist.

Satz 2.2 Sei $k=p$ prim und sei A eine Matrix der Form (2). Dann erzeugt A genau dann eine De-Bruijn-Folge der Ordnung $n$, wenn das charakteristische Polynom $p_{A}(x)$ von $A$ über $\mathbb{F}_{p}$ primitiv ist, d.h. wenn es irreduzibel ist und eine beliebige Nullstelle von $p_{A}(x)$ in $\mathbb{F}_{p^{n}}$ die multiplikative Gruppe des endlichen Körpers $\mathbb{F}_{p^{n}}$ erzeugt.

Die Aussage ist allgemein bekannt. Wir haben aber in der Literatur keine lückenlose Herleitung gefunden und geben deshalb der Vollständigkeit halber einen Beweis an. Wir bezeichnen den $n$-dimensionalen Vektorraum über dem endlichen Körper $\mathbb{F}_{p}$ mit $\left(\mathbb{F}_{p}\right)^{n}$ und identifizieren die Teilwörter der Länge $n$ analog zu (3) mit Vektoren in $\left(\mathbb{F}_{p}\right)^{n}$.

Beweis Nehmen wir zunächst an, dass $p_{A}(x)$ primitiv ist. Dann ist $p_{A}(x)$ insbesondere irreduzibel und hat $n$ verschiedene Nullstellen $\lambda_{1}, \ldots, \lambda_{n}$ in $\mathbb{F}_{p^{n}}$ (Satz 11.3 in [8]). Wir können $A$ also über $\mathbb{F}_{p^{n}}$ diagonalisieren, d.h. es existiert ein $U$ mit $A=U^{-1} D U$, und $D$ ist eine Diagonalmatrix mit den Diagonalelementen $\lambda_{1}, \ldots, \lambda_{n}$. Da $p_{A}(x)$ primitiv ist, haben alle $\lambda_{i}$ die maximale Ordnung $p^{n}-1$ (siehe dazu Satz 11.7 in [8]). Es gilt somit $A^{p^{n}-1}=E_{n}$, wobei $E_{n}$ die $n$-dimensionale Einheitsmatrix ist, und wir erhalten $A^{p^{n}-1} v=v$ für alle $v \in\left(\mathbb{F}_{p}\right)^{n} \backslash\left\{(0, \ldots, 0)^{T}\right\}$. Andererseits gilt aber $A^{k} v \neq v$ für $1 \leq k<p^{n}-1$ und $v \in\left(\mathbb{F}_{p}\right)^{n} \backslash\left\{(0, \ldots, 0)^{T}\right\}$, denn sonst hätte $A^{k}$ einen Eigenvektor zum Eigenwert 1, was aber nicht möglich ist, weil die Eigenwerte $\lambda_{i}$ alle maximale Ordnung haben.

Nehmen wir nun an, dass $A$ eine De-Bruijn-Folge im Sinne der Definition 2.1 erzeugt. Dann gibt es einen Vektor $v \in\left(\mathbb{F}_{p}\right)^{n} \backslash\left\{(0, \ldots, 0)^{T}\right\}$, so dass die Vektoren $v=$ $A^{0} v$ und $A^{1} v, \ldots, A^{p^{n}-2} v$ alle paarweise verschieden sind. Es folgt, dass $A^{p^{n}-1} v=$ $v$, denn sonst würde es ein $1 \leq k<p^{n}-1$ mit $A^{k} v=A^{p^{n}-1} v$ geben, was wegen der Invertierbarkeit von $A$ impliziert: $v=A^{p^{n}-1-k} v$. Entsprechend sehen wir, dass $A^{p^{n}-1} w=w$ für alle $w \in\left(\mathbb{F}_{p}\right)^{n} \backslash\left\{(0, \ldots, 0)^{T}\right\}$, woraus folgt, dass die Ordnung von $A$ gleich $p^{n}-1$ ist. Weil die Ordnung endlich und teilerfremd $\mathrm{zu} p$ ist, folgt die Diagonalisierbarkeit von $A$ über dem algebraischen Abschluss von $\mathbb{F}_{p}$. Weiter haben alle Eigenwerte von $A$ eine Ordnung, die $p^{n}-1$ teilt (der Beweis hierzu findet sich im Anhang). Wenn ihre Ordnung ein echter Teiler $t$ von $p^{n}-1$ wäre, gäbe es ein $v$ mit $A^{t} v=v$ und die Operation von $A$ auf $\left(\mathbb{F}_{p}\right)^{n}$ hätte mehr als eine Bahn. 
Das charakteristische Polynom von

$$
A=\left(\begin{array}{ccccc}
0 & 1 & 0 & \ldots & 0 \\
0 & 0 & 1 & \ldots & 0 \\
\vdots & \vdots & & \ddots & \vdots \\
0 & 0 & \ldots & & 1 \\
a_{n-1} & a_{n-2} & \ldots & \ldots & a_{0}
\end{array}\right) .
$$

hat die Form

$$
x^{n}-a_{0} x^{n-1}-a_{1} x^{n-2}-\ldots-a_{n-1} .
$$

Besonders gut einprägen lässt sich der Zaubertrick für Matrizen, bei denen das charakteristische Polynom sehr dünn besetzt ist. Für $p=2$ sind dies die Trinomials, d. h. Polynome, bei denen genau drei Koeffizienten von 0 verschieden sind. Primitive Trinomials über $\mathbb{F}_{2}$ existieren leider nicht für jeden $\operatorname{Grad} n$. Für $n \equiv 0 \bmod 8$ gibt es keine [12], ebenso für andere Grade, wie z. B. $n=13$ (siehe auch Kapitel 5 in [6]).

Die Anzahl der linearen De-Bruijn-Folgen für gegebenes $p$ und $n$ lässt sich einfach angeben.

Satz 2.3 Es gibt

$$
\frac{\varphi\left(p^{n}-1\right)}{n}
$$

lineare De-Bruijn-Folgen, wobei $\varphi$ die Eulersche $\varphi$-Funktion ist.

Beweis Die multiplikative Gruppe $\mathbb{F}_{p^{n}}^{*}$ von $\mathbb{F}_{p^{n}}$ ist zyklisch mit $p^{n}-1$ Elementen. Jede zyklische Gruppe hat $\varphi\left(p^{n}-1\right)$ erzeugende Elemente. Demzufolge gibt es $\varphi\left(p^{n}-1\right)$ primitive Elemente in $\mathbb{F}_{p^{n}}^{*}$. Jedes primitive Polynom hat $n$ paarweise verschiedene Nullstellen, die alle primitive Elemente sind. Somit gibt es $\frac{\varphi\left(p^{n}-1\right)}{n}$ normierte, primitive Polynome (vergleiche auch Abschnitt 11.10 in [8]). Zu jedem dieser Polynome lässt sich gemäß (4) eine zugehörige Matrix $A$ angeben, die dann eine lineare De-Bruijn-Folge beschreibt.

Wenn wir dies mit der Anzahl aller De-Bruijn-Folgen (siehe (1)) vergleichen, sehen wir, dass der Anteil der linearen Folgen an allen De-Bruijn-Folgen verschwindend gering ist.

\subsection{Teilfolgen linearer De-Bruijn-Folgen}

Nun hat nicht jedes gängige Kartenspiel $p^{n}$ Karten. Wenn wir den Trick beispielsweise für das übliche Pokerkartenspiel ohne Joker anwenden wollen, benötigen wir eine De-Bruijn-Folge mit 52 Elementen. Wir versuchen diese durch geschicktes Herausschneiden aus einer längeren Folge zu generieren. 
Betrachten wir dazu eine vollständige De-Bruijn-Folge $w$ der Länge $2^{n}$, die wir auf eine Folge der Länge $m<2^{n}$ kürzen wollen. Sei $b_{1} \ldots b_{n}$ ein Teilstück mit der Eigenschaft, dass $b_{1} \ldots b_{n-1}$ das erste Mal in $w$ auftritt. Insbesondere findet sich das Teilstück $b_{1} \ldots \overline{b_{n}}$ mit $\overline{b_{n}}=b_{n}-1$ erst später. Dann können wir $w$ kürzen, indem wir bereits bei diesem ersten Auftreten das Zeichen $b_{n}$ durch $\overline{b_{n}}$ ersetzen und dann mit der für die lineare Folge vorgegebenen Berechnungsvorschrift fortfahren.

Wir wollen nun zeigen, dass wir für jedes $m$ ein Teilstück $b_{1} \ldots b_{n}$ finden, so dass wir die Gesamtsequenz der Länge $2^{n}$ durch die oben beschriebene Methode auf eine Folge der Länge $m$ kürzen können.

Dafür machen wir uns klar, dass $A$ die Matrixdarstellung der Multiplikation mit $\alpha \in \mathbb{F}_{p^{n}}, p_{A}(\alpha)=0$ in $\left(\mathbb{F}_{p}\right)^{n}$ als $\mathbb{F}_{p}$-Vektorraum ist. Das können wir einfach nachrechnen, indem wir als Basis von $\left(\mathbb{F}_{p}\right)^{n}$ die Elemente

$$
\begin{aligned}
v_{1} & =\alpha^{n-1}-a_{0} \alpha^{n-2}-a_{1} \alpha^{n-3}-\ldots-a_{n-2}, \\
v_{2} & =\alpha^{n-2}-a_{0} \alpha^{n-3}-\ldots-a_{n-3}, \\
v_{3} & =\alpha^{n-3}-a_{0} \alpha^{n-4}-\ldots-a_{n-4}, \\
& \ldots \\
v_{n-1} & =\alpha-a_{0} \\
v_{n} & =1
\end{aligned}
$$

nehmen und somit $\left(\mu_{1}, \ldots, \mu_{n}\right)^{T}$ mit $\mu_{1} v_{1}+\ldots+\mu_{n} v_{n}$ identifizieren.

Wenn wir jetzt die Konvention treffen, dass wir mit $v=(0,0, \ldots, 1)^{T} \widehat{=} 1$ beginnen, entsprechen die nachfolgenden $n$-stelligen Wörter den Elementen $\alpha, \alpha^{2}, \ldots$. Sei $\alpha^{r}$ das Element, das dem ersten Auftreten des gewünschten Teilstücks entspricht, dann ist $\alpha^{r}-1$ das Element, bis zu dem wir herausschneiden wollen.

Satz 2.4 Für jedes $\widetilde{m} \in\left\{1, \ldots, p^{n}-1\right\}$ gibt es ein $r=r(\widetilde{m})$ mit

$$
\alpha^{r}=\alpha^{r+\widetilde{m}}+1
$$

Beweis Aus $\alpha^{r}-\alpha^{r+\widetilde{m}}=1$ folgt $\alpha^{r} \cdot\left(1-\alpha^{\widetilde{m}}\right)=1$. Somit ist $\alpha^{r}$ das Inverse von $\left(1-\alpha^{\widetilde{m}}\right)$ in $\mathbb{F}_{p^{n}}^{*}$.

Wir finden somit zu einer linearen De-Bruijn-Folge der Länge $p^{n}$ und einer beliebigen Zahl $0<\widetilde{m} \leq p^{n}-1$ eine Folge der Länge $m=p^{n}-\widetilde{m}$, die durch Kürzen aus der De-Bruijn-Folge hervorgeht und die Eigenschaft hat, dass jedes Wort der Länge $n$ maximal einmal (aber natürlich nicht unbedingt genau einmal) vorkommt.

Für $p>2$ hat man natürlich noch weitere Möglichkeiten, indem man das Teilwort $b_{1} \ldots b_{n}$ durch $b_{1} \ldots \widehat{b}_{n}$ mit $0 \leq \widehat{b}_{n} \leq p-1$ und $\widehat{b}_{n} \neq b_{n}$ ersetzt. 


\section{Anwendung in der Zauberei}

\subsection{Der Farbtrick mit 52 Karten}

In der autobiographischen Erzählung „Fooling Houdini“ des Zauberers Alex Stone beschreibt der Autor, wie er den in Abschn. 2.1 beschriebenen Zaubertrick auf das Pokerkartenspiel mit 52 Karten erweiterte [11]. Er ließ sich dazu ein Computerprogramm schreiben, das mittels Brute-Force-Suche eine geeignete Folge lieferte und lernte diese zusammen mit der Kodierung in Kartenwerte mit Mnemotechniken, die er eingehend in seinem Buch beschreibt, auswendig. In diesem Abschnitt erläutern wir eine Version für 52 Karten, die mit Rechnen modulo 2 und dem Auswendiglernen von lediglich 8 Ausnahmekarten auskommt.

Wir starten mit dem primitiven Polynom

$$
p_{A}(x)=x^{6}+x^{5}+1
$$

über $\mathbb{F}_{2}$ und erhalten die Folge mit 63 bzw. 64 Zeichen

(0)000001111110101011001101110110100100111000101111001010001100001.

Aus der Folge ohne die führende Null soll jetzt eine zusammenhängende Folge der Länge $\widetilde{m}=11$ weggeschnitten werden, um den Trick mit 52 Karten durchführen zu können.

Bemerkung 3.1 Betrachten wir zunächst die Idee aus Abschn. 2.3.

Sei $\alpha \in \mathbb{F}_{2^{6}}$ eine Nullstelle von $x^{6}+x^{5}+1$. Nach Satz 2.4 suchen wir ein $r$ mit

$$
\alpha^{r}=\left(1-\alpha^{\widetilde{m}}\right)^{-1} \text {. }
$$

Es gilt

$$
\left(1-\alpha^{\widetilde{m}}\right)^{-1}=\alpha^{5}+\alpha^{4}+\alpha^{3}+\alpha=\alpha^{14} \text { und } \alpha^{14}=\alpha^{14+11}+1 .
$$

Das Element $\alpha^{14}$ wird durch das sechsstellige Wort 101100 repräsentiert (beginnt an der 15. Stelle der obigen Folge). Hier schneiden wir nun aus (die Folge der Länge $\widetilde{m}=11$ ist fett gedruckt)

\section{1}

und erhalten damit eine Folge der Länge 52, bei der jedes sechsstellige Wort nur einmal auftritt. Leider enthält die verbleibende Folge nun 27 Nullen und 25 Einsen, so dass für die Anwendung des Zaubertricks weitere Anpassungen nötig sind. So kann man eine rote Ausnahmekarte, z. B. die Herz Dame, entgegen der Regel mit einer 0 identifizieren und zunächst einmal in Erfahrung bringen, ob jemand die Herz Dame hat. Falls dies nicht der Fall ist, kann man dann wieder alle roten Karten mit einer 
1 identifizieren. Im Folgenden gehen wir aber auf eine noch elegantere Methode ein, die wir speziell für den Fall mit $m=52$ Karten gefunden haben.

Wir suchen nun für diesen speziellen Fall eine zusammenhängende Folge der Länge 12, die wir herausschneiden können, so dass deren sechsstellige Teilwörter alle paarweise verschieden sind und die zudem genau 26 Nullen und 26 Einsen enthält. Es gibt genau drei mögliche Folgen mit diesen Eigenschaften (die Teilwörter der Länge 12 sind unten fettgedruckt):

0000011111101010110011011101101001001110001011110010100011000010, 0000011111101010110011011101101001001110001011110010100011000010 , 0000011111101010110011011101101001001110001011110010100011000010 ,

wovon die zweite und dritte auf die gleiche Folge der Länge 52 führen:

0000011111101010110011011101101001110010100011000010.

Diese Folge besitzt die schöne Eigenschaft, dass auch alle Zweiertupel 00, 11, 01 und 10 in gleicher Anzahl, also genau je 13 Mal auftreten und wir wie beim Trick mit den 32 Karten diese für die Kodierung des Kartentyps (00 für Kreuz, 01 für Pik, 10 für Herz und 11 für Karo) verwenden können.

Die Zuordnung von sechsstelligem Wort zu Spielkarte ist hier wegen der herausgeschnittenen Wörter etwas komplexer als im Fall mit 32 Spielkarten. Wir beschreiben eine mögliche Variante, bei der man sich möglichst wenig merken muss. Die letzten vier Zeichen sollen den Wert der Karte festlegen. Dabei gilt zunächst:

\begin{tabular}{cccccccc} 
letzte vier Ziffern & 0000 & 0011 & 0100 & 0101 & 0110 & 0111 & 1000 \\
Kartenwert & 2 & 3 & 4 & 5 & 6 & 7 & 8 \\
\hline letzte vier Ziffern & 1001 & 1010 & 1011 & 1100 & 1101 & 1111 & \\
Kartenwert & 9 & 10 & Bube & Dame & König & Ass &
\end{tabular}

Es gibt nun acht Karten, die in diesem Schema nicht erfasst sind. Das sind die Ausnahmen, die seperat gelernt werden müssen:

\begin{tabular}{cc} 
sechsstelliges Wort & Karte \\
\hline 000001 & Kreuz 5 \\
010001 & Pik 2 \\
100001 & Herz 4 \\
000010 & Kreuz 9 \\
110010 & Karo 8 \\
001110 & Kreuz Bube \\
101110 & Herz Ass \\
111110 & Karo Dame
\end{tabular}




\subsection{Ein Trick für $p=3$}

Wir betrachten das primitive Polynom $p_{A}(x)=x^{4}+2 x+2$ über $\mathbb{F}_{3}$. Die zugehörige De-Bruijn-Folge mit $81=3^{4}$ Zeichen lässt sich durch die Vorschrift $w_{1} w_{2} w_{3} w_{4} \mapsto$ $w_{2} w_{3} w_{4} w_{5}$ mit $w_{5} \equiv w_{1}+w_{2} \bmod 3$ erzeugen:

00010011012110021020122101011112220112120

0020022021220012010211202022221110221210.

Tatsächlich gibt es ein Kartenspiel mit 81 Karten, das dafür gerade optimal geeignet ist: das Kartenspiel „Set“. Wir erläutern kurz die Idee des Spiels, das eine interessante mathematische Struktur besitzt und deshalb schon Inhalt zahlreicher mathematischer Arbeiten war (siehe z. B. [3] für einen Übersichtsartikel).

Das Spiel besteht aus 81 Karten in den Farben Rot, Grün oder Blau, mit entweder einem, zwei oder drei Symbolen, die drei verschiedene Formen haben können. Die Symbole sind entweder nicht, ganz oder nur schraffiert ausgefüllt (vergleiche Abb. 1). Das bedeutet: Jede Karte ist durch vier Eigenschaften bestimmt, und zwar Anzahl, Füllung, Farbe und Form. Drei Karten bilden ein Set, wenn für jede der vier Eigenschaften gilt, dass entweder alle drei Karten die gleiche Ausprägung oder paarweise verschiedene Ausprägungen aufweisen. Die Abbildung zeigt drei Beispiele:
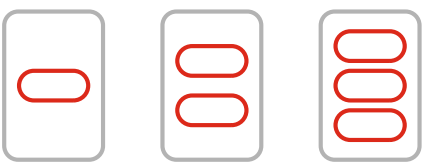

Set
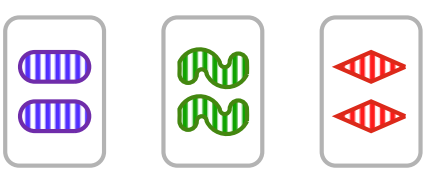

Set
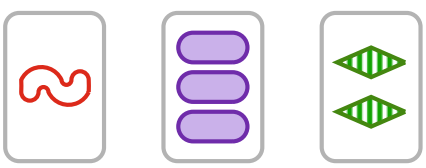

Set
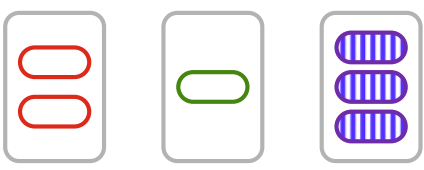

kein Set

Im ersten Fall stimmen Farbe, Form und Füllung überein. Die Anzahl ist immer unterschiedlich. Im zweiten Fall stimmen Anzahl und Füllung auf allen drei Karten überein, Form und Farbe weisen paarweise unterschiedliche Ausprägungen auf. Beim nächsten Set liegen alle vier Eigenschaften in unterschiedlichen Ausprägungen 


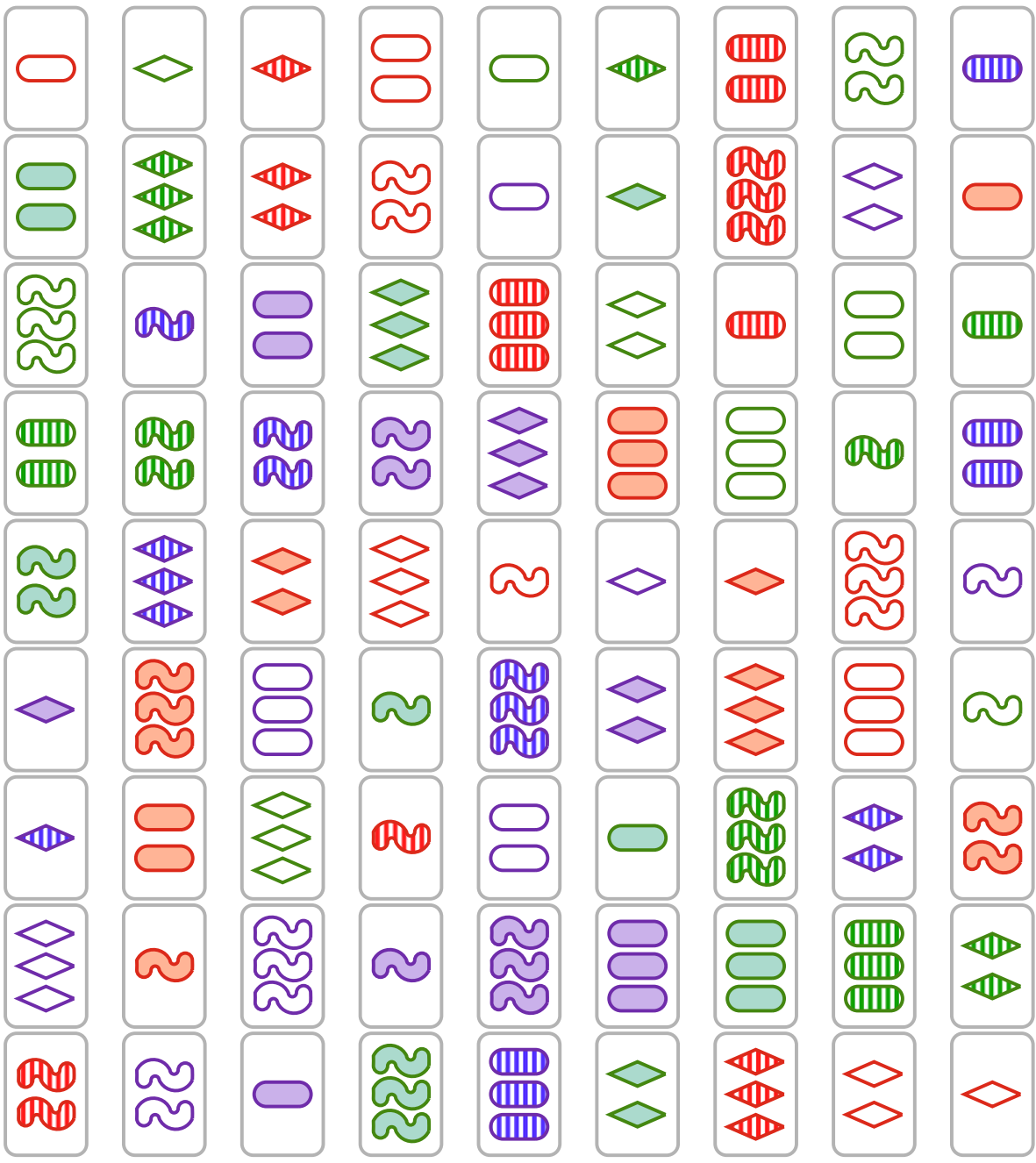

Abb. 1 Eine Konfiguration des Set-Kartenspiels, so dass die Anzahlen der abgebildeten Symbole von vier aufeinander folgenden Karten die Karten eindeutig spezifizieren

vor. Die letzten drei Karten bilden kein Set. Die Füllung ist weder überall identisch, noch paarweise verschieden.

Um zu spielen, werden die Karten gemischt und 12 Karten bildoben auf den Tisch gelegt. Alle Mitspieler suchen nun gleichzeitig ein Set. Wer ein Set gefunden hat, erhält die drei Karten. Diese werden anschließend durch neue Karten vom Stapel ersetzt. Wer am Ende die meisten Sets gefunden hat, gewinnt das Spiel.

Nicht immer gibt es unter den 12 aufgedeckten Karten ein Set. In diesem Fall wird nach und nach eine weitere Karte dazugenommen, bis jemand ein Set findet. Pellegrino zeigte, dass erst bei 21 Karten die Existenz eines Sets garantiert ist [10].

Mit De-Bruijn-Folgen können wir jetzt einen Mentaltrick für das Kartenspiel „Set“ beschreiben. Jeder Karte lässt sich also kanonisch eine vierstellige Zahl im 
Dreiersystem zuordnen. Die erste Ziffer steht dabei beispielsweise für die Anzahl (weniger 1), die zweite für die Füllung (0 für keine, 1 für schraffiert und 2 für ganz), die dritte für die Farbe (0 für rot, 1 für grün und 2 für blau) und die letzte für die Form (0 für die Raute, 1 für das Oval und 2 für die geschwungene Form).

Wenn der Kartenstapel entsprechend der De-Bruijn-Folge in (5) gelegt ist (vergl. Abb. 1) und vier Zuschauer nacheinander eine Karte abgehoben haben, sind die Karten allein durch die Anzahl der Symbole eindeutig bestimmt.

Anmerkung Zur Erstellung der Abbildungen wurden die Skatkarten-Grafiken unter https://de.wikipedia.org/wiki/Datei:Svg-cards-2.0.svg bzw. https://tex. stackexchange.com/questions/207929/how-to-draw-the-cards-of-a-deck und die Grafiken zum Spiel ,Set“ unter https://ctan.org/tex-archive/graphics/pgf/contrib/ setdeck verwendet (beides zuletzt aufgerufen am 25.11.2020).

Funding Open Access funding enabled and organized by Projekt DEAL.

Open Access Dieser Artikel wird unter der Creative Commons Namensnennung 4.0 International Lizenz veröffentlicht, welche die Nutzung, Vervielfältigung, Bearbeitung, Verbreitung und Wiedergabe in jeglichem Medium und Format erlaubt, sofern Sie den/die ursprünglichen Autor(en) und die Quelle ordnungsgemäß nennen, einen Link zur Creative Commons Lizenz beifügen und angeben, ob Änderungen vorgenommen wurden.

Die in diesem Artikel enthaltenen Bilder und sonstiges Drittmaterial unterliegen ebenfalls der genannten Creative Commons Lizenz, sofern sich aus der Abbildungslegende nichts anderes ergibt. Sofern das betreffende Material nicht unter der genannten Creative Commons Lizenz steht und die betreffende Handlung nicht nach gesetzlichen Vorschriften erlaubt ist, ist für die oben aufgeführten Weiterverwendungen des Materials die Einwilligung des jeweiligen Rechteinhabers einzuholen.

Weitere Details zur Lizenz entnehmen Sie bitte der Lizenzinformation auf http://creativecommons.org/ licenses/by/4.0/deed.de.

\section{Anhang}

Es ist bekannt, dass Matrizen endlicher Ordnung über den komplexen Zahlen diagonalisierbar sind. Deutlich seltener findet man in der Literatur den Fall, dass der zugrundeliegende Körper positive Charakteristik hat, also beispielsweise der algebraische Abschluss eines endlichen Körpers ist. Deshalb geben wir hier der Vollständigkeit halber einen Beweis für das folgende Resultat.

Satz 4.1 Sei A eine $n \times n$-Matrix über dem endlichen Körper $\mathbb{F}_{p}$ mit $A^{k}=E_{n}$ und $k \neq \equiv 0 \bmod p$. Dann lässt sich $A$ über dem algebraischen Abschluss von $\mathbb{F}_{p}$ diagonalisieren. Die Eigenwerte haben eine Ordnung, die $k$ teilt.

Beweis Für jede Matrix $A$ existiert über dem algebraischen Abschluss eine Darstellung der Form

$$
A=U^{-1} J \mathrm{U}
$$


mit einer Jordan-Matrix

$$
J=\left(\begin{array}{ccc}
J_{n_{1}}\left(\lambda_{1}\right) & & 0 \\
& \ddots & \\
0 & & J_{n_{j}}\left(\lambda_{j}\right)
\end{array}\right)
$$

mit den $n_{i} \times n_{i}$-Jordan-Blöcken

$$
J_{n_{i}}\left(\lambda_{i}\right)=\left(\begin{array}{cccc}
\lambda_{i} & 1 & & 0 \\
& \lambda_{i} & \ddots & \\
& & \ddots & 1 \\
0 & & & \lambda_{i}
\end{array}\right), 1 \leq i \leq j \text { und } n_{1}+\ldots+n_{j}=n .
$$

Aus $A^{k}=E_{n}$ folgt $J^{k}=E_{n}$, also $J_{n_{i}}^{k}=E_{n_{i}}$. Nehmen wir nun an, dass $n_{i}>1$ für ein $i$. Dann ergibt sich aus

$$
J_{n_{i}}^{k}\left(\lambda_{i}\right)=\left(\begin{array}{cccc}
\lambda_{i}^{k} & \left(\begin{array}{c}
k \\
1
\end{array}\right) \lambda_{i}^{k-1} & \ldots & \left(\begin{array}{c}
k \\
n_{i}-1
\end{array}\right) \lambda_{i}^{k-n_{i}+1} \\
& \ddots & \ddots & \vdots \\
& & \ddots & \left(\begin{array}{c}
k \\
1
\end{array}\right) \lambda_{i}^{k-1} \\
0 & & & \lambda_{i}^{k}
\end{array}\right)
$$

und $\lambda_{i} \neq 0$, dass der Binomialkoeffizient $\left(\begin{array}{c}k \\ 1\end{array}\right)$ gleich 0 ist. Dies ist aber nur möglich, falls $k \equiv 0 \bmod p$.

Somit gilt $n_{i}=1$ für alle $i$ und die Matrix $A$ ist diagonalisierbar.

\section{Literatur}

1. van Aardenne-Ehrenfest, T., de Bruijn, N.G.: Circuits and trees in oriented linear graphs. Simon Stevin 28, 203-217 (1951)

2. Behrends, E.: Zaubern und Mathematik. Springer, Berlin, Heidelberg, New York (2017) https://doi.org/ 10.1007/978-3-658-17505-4

3. Davis, B.L., Maclagan, D.: The card game SET. Math Entertain 25(3), 33-40 (2003). https://doi.org/ 10.1007/BF02984846

4. Diaconis, P., Graham, R.: Magical Mathematics. Princeton University Press, Princeton (2012) https:// doi.org/10.1515/978-1-400-83938-4

5. Fredricksen, H.: A survey of full length nonlinear shift register cycle algorithms. SIAM Rev 24(2), 195-221 (1982)

6. Golomb, S.: Shift Register Sequences, 3. Aufl. World Scientific, Singapur (2017) https://doi.org/10. $1142 / 9361$

7. Jetzek, U.: Galois Fields, Linear Feedback Shift Registers and Their Applications. Carl Hanser, München (2018) https://doi.org/10.3139/978-3-446-46619-7

8. Kurzweil, H.: Endliche Körper - Verstehen, Rechnen, Anwenden, 2. Aufl. Springer, Berlin, Heidelberg, New York (2008) https://doi.org/10.1007/978-3-540-79598-8

9. Martin, M.H.: A problem in arrangements. Bull. Amer. Math. Soc. 40(12), 859-864 (1934)

10. Pellegrino, G.: Sul massimo ordine delle calotte in $S_{4,3}$. Matematich 25, 149-157 (1971) 
11. Stone, A.: Fooling Houdini: Magicians, Mentalists, Math Geeks, and the Hidden Powers of the Mind. Harper, New York City (2012)

12. Swan, R.G.: Factorization of polynomials over finite fields. Pacific J. Math. 12, 1099-1106 (1962) 